\title{
A hypothesis for primary open angle glaucoma, normal tension glaucoma and ocular hypertension.
}

\author{
Bhartendu Shukla* \\ Department of Ophthalmology, RJN ophthalmic Institute, Gwalior, India
}

\begin{abstract}
There will be only few clinical conditions in ophthalmology like glaucoma where nationally and internationally intensive and extensive research is going on toward the clinical conditions.
\end{abstract}

Keywords: Glaucoma, ocular hypertension, hypothesis, organism, intra-ocular pressure.

Accepted on February 08, 2018

\section{Introduction}

In spite of this we have yet to evolve a satisfactory definition of glaucoma which would explain all the different clinical conditions which are associated with this term. In text books, journals and Pubmed more than a dozen definitions are given. This in itself shows the complexity of the condition. In the present paper an effort has been made to correlate three important related entities, Primary Open Angle Glaucoma (POAG), Normotensive Glaucoma (NTG) and Ocular Hypertension (OHT) by a single hypothesis. Justification of lowering IOP in all these three conditions is validated.

\section{Basic Presumptions}

The following is a universally accepted principle which the Infection is directly proportional to the virulence of the organism or Infection is inversely proportional to the resistance of the organism Thus it can be stated that Infection $=$ Virulence / Resistance (or) Disease $=$ Virulence $/$ Resistance .

\section{Historical Background}

From a very early period glaucoma has been recognized as a hard eye or an eye with raised intra-ocular pressure (above normal range 9-21 $\mathrm{mm} \mathrm{Hg}$ ) [1-2] resulting in pain and gradual irreversible blindness in elderly persons. Till almost at the end of $20^{\text {th }}$ century the most acceptable cause of glaucoma was a raised intra-ocular pressure. This was further confirmed by the fact that by lowering the intra-ocular pressure medically or surgically there was a definite relief from pain and the vision was prevented from further deterioration. Thus glaucoma and raised IOP were considered almost as synonyms. The normal IOP was considered as $17 \mathrm{~mm} \mathrm{Hg}$ as taken by Schiotz tonometer varying from 13-21 mm Hg. Any pressure above $21 \mathrm{~mm} \mathrm{Hg}$ could be considered as indicative of glaucoma. However with a planation tonometer $15 \mathrm{~mm} \mathrm{Hg}$ was considered normal. It was further noted that IOP is also influenced by corneal thickness [3]. The role of blood supply to the optic disc has been emphasized by many workers [46]. The first big jolt to this hypothesis came from the fact that many workers showed that even within this range of normal intraocular pressure there could be damage to the optic nerve head resulting in loss of vision/visual fields. This was termed Normotensive glaucoma which showed typical glaucomatous fields and disc changes [7]. Thus the main focus of attention in glaucoma shifted from raised IOP to chronic progressive damage to optic nerve head. It was objectively evaluated by the optic disc changes and subjectively by the regression of visual fields measured both by static and kinetic methods. The second big jolt came from the fact that many individuals with fairly high IOP did not get any optic nerve head damage as recorded by perimetry and optic disc study. Thus raised IOP further lost relevance in the pathogenesis of glaucoma and it was relegated a low status of a risk factor. This condition is described as Ocular Hypertension (OHT) and is strictly not a part of Glaucoma Complex [8] Now glaucoma is considered as chronic progressive optic nerve head damage with typical visual field changes and disc changes. In its pathogenesis a raised IOP is only a risk factor among many other risk factors. Thus we find that during the last hundred years the status of IOP has come down from Hero to almost Zero as regards the pathogenesis of glaucoma [9].

\section{Present Hypothesis}

However a new hypothesis is being suggested for two reasons. Firstly, on the theoretical grounds we have downgraded the role of IOP in the pathogenesis of glaucoma as mentioned earlier. However on the practical side what are we doing to treat glaucoma except by lowering of IOP medically or surgically all big companies are competing to make a drug which would be very effective in lowering IOP without much side effects. They are making various combinations of drugs for the same purpose. Combination of drugs and tablets are being explored for this very purpose. Laser therapy has also the same end in view. All surgical techniques are looking for effectively controlling the IOP Trabeculectomy is blamed not keeping IOP low for a long time. So how do we correlate these two diametrically opposite philosophies there is considerable talk on neuron-protection. Theoretically it is a good proposition but what concrete has been found till date except that Brimonidine has shown some utility in animal model [10] we talk of optic nerve damage. Optic nerve is nothing but the axons of the ganglion cells of the retina. So the primary seat of damage is probably in the ganglion cells of the retina though we clinically see this damage more clearly at the optic disc and its adjacent areas. Do we have any method to quantify Ganglion Cell Viability (GCV) probably not yet I would like to return to the initial hypothesis

Disease $=$ Virulence $/$ Resistance 
This formula can be modified as follows Glaucoma $=$ Height of IOP / Ganglion cell viability.

\section{Discussion}

Ganglion cell viability could be taken as the resistance of retina to the damage caused by raised IOP. This would offer a viable explanation to various models of primary glaucoma. For sake of understanding we can imagine that the value of GCV can also be graded like IOP. It may be very high, high, normal, low or very low. Primary Open angle glaucoma would result when IOP is high or very high and GCV is normal or low. Normal tension glaucoma would result when IOP is normal or low and GCV is low or very low. Similarly ocular hypertension would result when IOP is high or very high but GCV is also high or very high. Thus in every situations we find that IOP plays an important role. No damage is done in ocular hypertension because GCV is also equally high. Damage is done in low IOP because GCV is equally low so we find that in every situation height of IOP plays an important role. That its results appear variable is due to variability in the accompanying value of GCV Further proof of this theory is that some cases of ocular hypertension do turn into glaucoma and even in Normal Tension glaucoma further lowering IOP is found useful. We have only qualitatively shown the value of GCV the crux of the matter is to work out a reliable quantitative value of GCV like IOP and if the association found is correct this theory would prove its validity. The earliest mechanical theory of primary glaucoma cannot be refuted. However subsequently several authors have postulated the vascular theory [7] This hypothesis also reconciles the validity of lowering IOP in almost all cases of glaucoma as we are actually doing and it also establishes the value of IOP in the causation and management of glaucoma. However the second factor GCV also needs further research for its validity. Some of the factors which determine the GCV include heredity, ganglion cell layer thickness, vascularity of optic nerve head indicated by peripapillary, choroidal layer thickness and diameter of the peripapillary retinal blood vessels. From these parameters a single parameter, i.e. ganglion cell viability can be worked out which is being attempted. In this context abiotrophy (pre mature death or degeneration of tissues) can also be an important factor [11]. This can explain heredity, bilaterally and early onset of glaucoma in some cases.

\section{Conclusion}

In spite of extensive work on glaucoma all over the world this condition is still evading its exact pathogenesis and many aspects of this condition are still not very clear. Recently, Hasnain [12] that optic disc may be sinking in chronic glaucoma. The significance of IOP in glaucoma has gone down considerably due to the discovery of normal tension glaucoma and ocular hypertension. In spite of this the main treatment remains lowering of IOP medically and or surgically. An entity Ganglion Cell Viability (GCV) is conceived which could be an important factor in offering a viable basis for lowering IOP not only in Primary Open Angle glaucoma but also in Low Tension Glaucoma and Ocular Hypertension. Quantitative estimation of GCV is an important issue and may further justify the present hypothesis.

\section{Conflict of Interest}

The author declares no conflict of interest.

\section{References}

1. Norman DC, Yoshikawa TH. In Infectious diseases of the aging A clinical hand book.2001:1-336.

2. Healy PR, Thomas R. In: Fast Facts Glaucoma Healy.2010:1-9.

3. Conception A. Determination of corneal thickness and intraocular pressure, In Highlights of Ophthalmology, Indian Edition.2012;40:11-2.

4. Henknd P. Investigataive OIphthal.1967;6:103.

5. Hayreh SS. Pathogenesis of visual field defect role of biliary circulation. British J Ophthalmol.1970;54:289.

6. Liberman MF, Drake MV, Stamper RL. In Becker Schaffer's Diagnosis and Therapy of Glaucomas. 1999:239.

7. Chandler PA, Grant WM., Ocular Hypertension Open Angle glaucoma, Arch. Ophthamol.1977;95:1-588.

8. Liberman MF, Drake MV, Stamper RL. In Becker-Schaffer's Diagnosis and Therapy of Glaucomas.1999;24:380.

9. Frisen L, Claesson M. Journal of Ophthalmology. 1984;91:1342-6.

10. Jonas JB, Naumann GO. Invest Ophthalmol. 1989;30:1604-11.

11. Rader J, Anderson DR. Amer J Ophthalmol. 1994;117:72-82

12. Hasnain SS. Optic disc may be sinking in chronic glaucoma, Ophthamology.2010;8:212-28.

\section{*Correspondence to:}

Bhartendu Shukla*
Department of ophthalmology,
RJN Ophthalmic Institute,
Gwalior,
India
Tel: +91 7512423351
E-mail: bhartendushukla@yahoo.com

